Ann. Sci. forest., 1979, 36 (4), 347-351.

NOTE TECHNIQUE

\title{
L'utilisation du torsiomètre en forêt : influence de l'opérateur et de l'appareil
}

\author{
G. NEPVEU \\ Station de Recherches sur la Qualité des Bois \\ Centre national de Recherches forestières, I.N.R.A., \\ Champenoux, 54280 Seichamps
}

\section{Résumé}

Cet article met en valeur l'intérêt de l'utilisation du couple de torsion pour retenir les arbres de densité élevée.

Il confirme l'influence très significative de l'instrument utilisé et de l'opérateur sur les valeurs du couple de torsion. Cependant, le classement des arbres est indépendant de ces deux sources de variation.

\section{Introduction}

L'emploi d'un torsiomètre pour apprécier la qualité du bois en forêt devient assez courant. On connaît les liaisons étroites existant entre le couple de torsion et diverses caractéristiques mécaniques ou physiques (densité en particulier). A ce sujet, Polge et Keller ont procédé en 1970 à une étude très complète qui a, en outre, précisé les modalités pratiques de mesure du couple, notamment l'influence des opérateurs et des appareils utilisés $(H$. Polge, R. Keller : Première appréciation de la qualité du bois en forêt par utilisation d'un torsiomètre. Annales des Sciences Forestières, 1970, 27 (2), 197-223.)

Ce dernier point nous a particulièrement préoccupé ; il serait grave, en effet, que la sélection pour des bois de forte densité opérée à l'aide d'un torsiomètre donne un classement des arbres dépendant du technicien procédant à la mesure, ou même de l'instrument qu'il manie. C'est dans cet esprit que nous avons procédé à l'expérience simple décrite ci-après.

\section{1. - Matériel et méthodes}

L'étude a été réalisée sur de jeunes Douglas de 15 à $30 \mathrm{~cm}$ de diamètre.

Dès l'abord, nous étions limité par le nombre de mesures par arbre. En effet, afin d'éviter les interactions dues aux trous de sondage, il nous fallait espacer ceux-ci 
d'au moins $10 \mathrm{~cm}$. Par ailleurs, l'éventuelle action de l'orientement devait être prise en compte en procédant aux mesures sur une même génératrice. Enfin, il y avait lieu de se préoccuper de la hauteur de mesure; on sait que la densité du bois (donc le couple) varie le long du tronc; en outre, l'opérateur ne peut travailler valablement que s'il repose sur le sol. Pratiquement, nous ne pouvions dépasser dix à douze valeurs de couple par arbre.

Trois opérateurs ont participé à l'expérience. Ils disposaient de deux torsiomètres et de deux tarières. Les torsiomètres ont été appliqués chacun sur la même tarière tout au long des mesures, en sorte qu'on comparera le couple 1 : (tarière $1+$ torsiomètre 1 ) au couple 2 : (tarière $2+$ torsiomètre 2 ) sans pouvoir faire la part des rôles de l'un et l'autre de ces instruments.

Les répétitions sont au nombre de deux. Sur chaque tige, chaque mesureur prenait donc le couple de torsion quatre fois (deux tarières $\times$ deux répétitions) ; nous avions tiré au sort l'endroit de mesure entre douze points espacés de $10 \mathrm{~cm}$ sur une génératrice située selon le même orientement pour tous les arbres. Les points en question s'échelonnaient de $40 \mathrm{~cm}$ à $1,50 \mathrm{~m}$ de hauteur.

Quinze arbres ont ainsi fait l'objet de mesures. Ces dernières ont été réalisées selon la modalité «stabilisé profond » en tenant compte de l'épaisseur de l'écorce.

II va sans dire que chaque opérateur ignorait les valeurs trouvées par les deux autres. En outre, pour chacun d'entre eux, on espaçait dans le temps les mesures effectuées sur le même arbre.

Le dispositif est parfaitement orthogonal.

\section{2. - Résultats}

Les cent quatre-vingts données figurent en Annexe.

\section{1. - Comparaison de la qualité des mesures des trois observateurs}

Dans un premier temps, nous avons songé à effectuer les analyses de variance observateur par observateur. L'intérêt d'un tel procédé réside dans le fait que la comparaison des variances résiduelles obtenues permet de voir s'il existe un (des) observateur meilleur ou moins bon que les $\left(\mathrm{I}^{\prime}\right)$ autres. En effet, une «erreur 》 trop forte indiquerait une répétabilité médiocre des mesures.

Le tableau 1 montre que, même s'il y a un effet très significatif dû à l'instrument de mesure (en fait à l'ensemble tarière + torsiomètre), l'interaction arbre $\times$ tarière n'est pas significative. Ceci signifie que le classement des arbres pour le couple sera le même si l'on emploie l'un ou l'autre des appareils.

Le rapport de la variance «erreur » la plus forte sur la variance « erreur » la plus faible conduit à un $\mathrm{F}$ calculé de $135 / 102=1,32$ pour un $\mathrm{F}$ calculé à 30 et 30 degrés de liberté de 2,39 (seuil 1 p. 100). On peut donc affirmer que la qualité des mesures des observateurs est identique. 
TABLEAU 1

Observateur A (measurer)

\begin{tabular}{|c|c|c|c|c|}
\hline $\begin{array}{l}\text { Sources de variation } \\
\text { (Sources of variation) }\end{array}$ & SCE & $\begin{array}{l}\text { Degrés } \\
\text { de liberté } \\
\text { (Degrees of } \\
\text { freedom) }\end{array}$ & $\begin{array}{l}\text { Carrés } \\
\text { moyens } \\
\text { (mean } \\
\text { squares) }\end{array}$ & $\begin{array}{c}\text { F calculés } \\
\text { (F calculated) }\end{array}$ \\
\hline $\begin{array}{l}\text { Arbre }(\text { Tree } \ldots \ldots \ldots \ldots \ldots \ldots \\
\text { Tarière (Increment borer) } \ldots \ldots \ldots \ldots \\
\text { Arbre } x \text { tarière (Tree } \times \text { increment } \\
\quad \text { borer) } \ldots \ldots \ldots \ldots \ldots \ldots \ldots \ldots \ldots \ldots \ldots \ldots \ldots \\
\text { Erreur (Error) } \ldots \ldots \ldots \ldots \ldots \ldots \ldots \ldots\end{array}$ & $\begin{array}{r}32084 \\
5511 \\
833 \\
3088\end{array}$ & $\begin{array}{r}14 \\
1 \\
14 \\
30\end{array}$ & $\begin{array}{r}2292 \\
5511 \\
60 \\
103\end{array}$ & $\begin{array}{l}22,25 * * \\
53,50 * * \\
0,58<1\end{array}$ \\
\hline Total $($ Total) $\ldots \ldots \ldots \ldots \ldots \ldots \ldots$ & 41516 & 59 & & \\
\hline
\end{tabular}

Observateur B

Sources de variation

SCE

23298
7041
1759
4050

36148

\section{Degrés \\ de liberté}

14
1
14
30
59

\section{Carrés \\ moyens}

1664

7041

125

135
F calculés

$12,33 * *$

$52,16 * *$

$0,93<1$

\section{Observateur C}

\begin{tabular}{|c|c|c|c|c|}
\hline Sources de variation & SCE & $\begin{array}{l}\text { Degrés } \\
\text { de liberté }\end{array}$ & $\begin{array}{l}\text { Carrés } \\
\text { moyens }\end{array}$ & F calculés \\
\hline 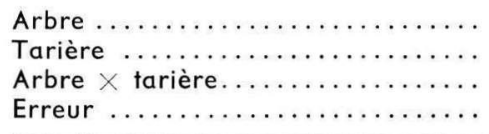 & $\begin{array}{r}19449 \\
9754 \\
990 \\
3062\end{array}$ & $\begin{array}{r}14 \\
1 \\
14 \\
30\end{array}$ & $\begin{array}{r}1389 \\
9754 \\
71 \\
102\end{array}$ & $\begin{array}{l}13,62 * * \\
95,63 * * \\
0,69<1\end{array}$ \\
\hline Total $\ldots \ldots \ldots \ldots \ldots \ldots \ldots$ & 33255 & 59 & & \\
\hline
\end{tabular}

\section{2. - Etude de l'effet «observateur » et des interactions qui s'y rattachent}

Le dispositif nous permet de faire la part des trois facteurs « arbre », " tarière » et «observateur », ainsi que des interactions d'ordre 2 et 3 existant entre ces effets.

Le tableau 2 d'analyse de la variance met en évidence des effets très significatifs des trois variables étudiées. En revanche, les diverses interactions conduisent toutes à des $F$ très éloignés des valeurs-seuils. Ceci prouve en particulier que le classement des arbres pour le couple ne va pas varier suivant l'opérateur ef la tarière. 
TABLEAU 2

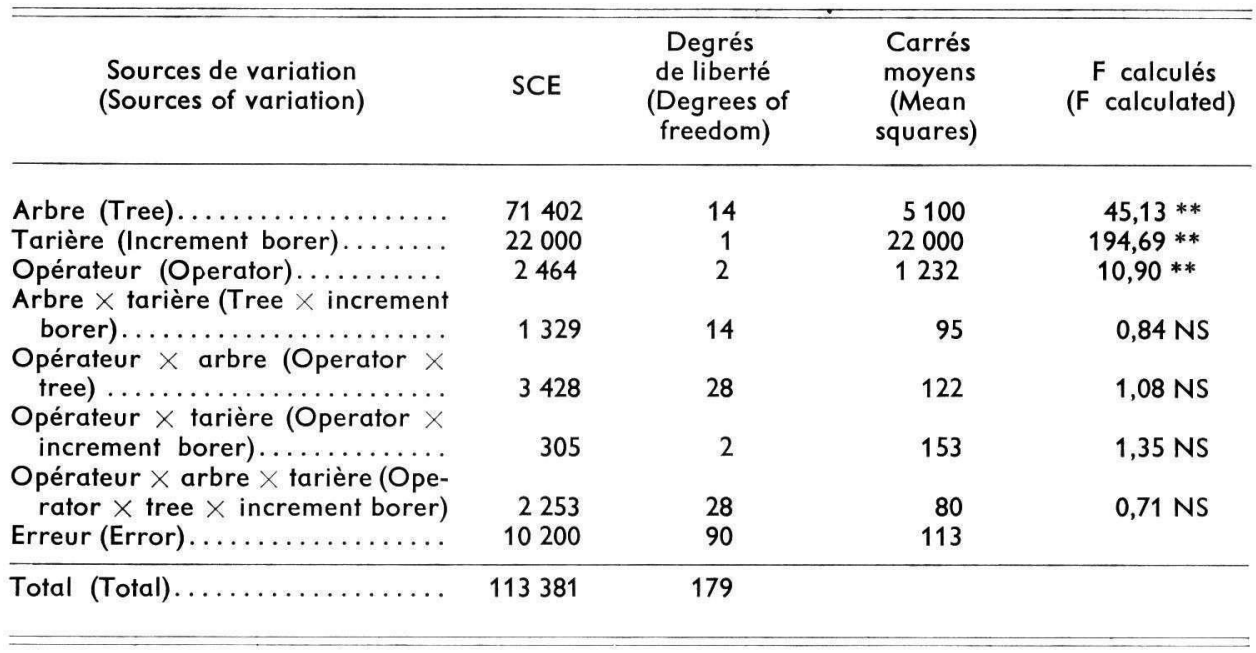

\section{Conclusion}

L'étude entreprise ci-dessus confirme l'influence très significative, à la fois de l'instrument utilisé et de l'opérateur, sur les valeurs du couple de torsion. Elle met en valeur l'intérêt d'un appareil qui pourrait éliminer ces sources de variation.

Cependant, on peut affirmer que, quel que soit l'appareil ou l'observateur, le classement des arbres pour le couple de torsion sera le même. C'est là un résultat encourageant pour les sélectionneurs de certaines essences qui utilisent la valeur du couple de torsion, dont la mesure sur le terrain est fort simple et rapide, pour retenir les arbres de densité élevée.

On se gardera cependant d'une application abusive de la méthode en se souvenant que l'époque de mesure (en sève ou hors sève), si elle n'intervient pas pour les conifères (Polge et Keller, 1970, op. cit.), influe de façon non négligeable sur les résultats observés pour le hêtre (R. Keller, F. Le Tacon, J. Timbal : la densité du bois de hêtre dans le nord-est de la France. Influence des caractéristiques du milieu et du type de sylviculture. Annales des Sciences Forestières, 1976, 33 (1), 1-17).

Reçu pour publication en février 1979.

\section{Zusammenfassung}

Die Verwendung des Torsiometers in Wald : Einfluss des Fachmanns und des Apparats

Dieser Beitrag betont das Interesse der Verwendung des Torsionsmoments, um die Baüme mit hoher Dichte zurückzuhalten.

Es bestätigt den sehr signifikanten Einfluss des verwendeten Apparats und des Fachmanns auf die Werte des Torsionsmoments. Die Klassifizierung der Baüme ist troztdem von diesen zwei Variationsquellen unabhängig. 


\section{Summary}

The torsiometer use «in situ »: influences of operator and instrument

This paper shows the interest of the use of torsion torque to select trees with high wood density.

It confirms the very significant influences of instrument and operator on the values of torsion torque. However, the classification of trees does not depend on these two sources of variation.

\section{ANNEXE}

Valeurs du couple en cm.kg selon arbre, observateur ef tarière

Values of torsion torque $(\mathrm{cm} . \mathrm{kg})$ in ferms of tree, measurer and increment borer

\begin{tabular}{|c|c|c|c|c|c|c|c|c|c|c|c|c|c|c|c|c|}
\hline \multirow{2}{*}{$\begin{array}{l}\text { Obser- } \\
\text { vateurs }\end{array}$} & \multirow{2}{*}{ Tarières } & \multicolumn{15}{|c|}{ Arbres } \\
\hline & & 1 & 2 & 3 & 4 & 5 & 6 & 7 & 8 & 9 & 10 & 11 & 12 & 13 & 14 & 15 \\
\hline \multirow{4}{*}{ A } & \multirow{2}{*}{ Tarière 1} & 175 & 170 & 220 & 210 & 170 & 165 & 145 & 145 & 195 & 150 & 200 & 180 & 180 & 150 & 155 \\
\hline & & 185 & 175 & 230 & 220 & 165 & 160 & 140 & 160 & 170 & 165 & 170 & 160 & 195 & 150 & 170 \\
\hline & \multirow{2}{*}{ Tarière 2} & 160 & 170 & 225 & 180 & 150 & 145 & 130 & 140 & 155 & 135 & 155 & 145 & 165 & 135 & 135 \\
\hline & & 165 & 165 & 210 & 220 & 130 & 145 & 130 & 120 & 150 & 145 & 160 & 140 & 175 & 135 & 135 \\
\hline \multirow{4}{*}{ B } & \multirow{2}{*}{ Tarière 1} & 185 & 180 & 195 & 235 & 165 & 180 & 150 & 140 & 170 & 175 & 195 & 165 & 205 & 155 & 165 \\
\hline & & 190 & 195 & 235 & 210 & 160 & 165 & 145 & 170 & 195 & 175 & 195 & 180 & 180 & 155 & 160 \\
\hline & \multirow{2}{*}{ Tarière 2} & 150 & 160 & 205 & 170 & 150 & 140 & 130 & 135 & 170 & 135 & 155 & 150 & 180 & 150 & 150 \\
\hline & & 175 & 145 & 210 & 200 & 160 & 160 & 125 & 140 & 155 & 140 & 165 & 145 & 180 & 155 & 135 \\
\hline \multirow{4}{*}{ C } & \multirow{2}{*}{ Tarière 1} & 195 & 175 & 210 & 210 & 175 & 180 & 160 & 160 & 205 & 170 & 210 & 180 & 215 & 160 & 180 \\
\hline & & 195 & 200 & 235 & 215 & 145 & 180 & 165 & 160 & 200 & 180 & 200 & 185 & 200 & 160 & 185 \\
\hline & \multirow{2}{*}{ Tarière 2} & 165 & 160 & 205 & 200 & 130 & 170 & 130 & 150 & 170 & 165 & 155 & 180 & 170 & 155 & 145 \\
\hline & & 165 & 160 & 190 & 175 & 140 & 150 & 140 & 145 & 170 & 145 & 180 & 160 & 165 & 135 & 155 \\
\hline
\end{tabular}

